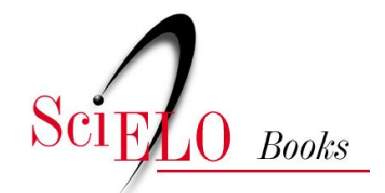

\title{
Capítulo 1 - Luchas sociales por el agua y el proceso de democratización en América Latina
}

\author{
José Esteban Castro
}

\section{SciELO Books / SciELO Livros / SciELO Libros}

CASTRO, J. E. Luchas sociales por el agua y el proceso de democratización en América Latina. In: Água e democracia na América Latina [online]. Campina Grande: EDUEPB, 2016, pp. 10-20. ISBN 978-85-7879-486-6. Available from: doi: 10.7476/9788578794866.0002. Also available in ePUB from: http://books.scielo.org/id/tn4y9/epub/castro-9788578794866.epub.

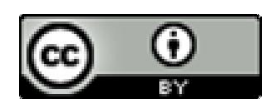

All the contents of this work, except where otherwise noted, is licensed under a Creative Commons Attribution $\underline{4.0 \text { International license. }}$

Todo o conteúdo deste trabalho, exceto quando houver ressalva, é publicado sob a licença Creative Commons Atribição 4.0.

Todo el contenido de esta obra, excepto donde se indique lo contrario, está bajo licencia de la licencia Creative Commons Reconocimento 4.0. 


\section{Capítulo 1}

\section{Luchas sociales por el agua y el proceso de democratización en América Latina ${ }^{1}$}

\section{Introducción}

En este artículo me propongo explorar algunas de las conexiones entre las luchas sociales por el agua y el ejercicio de la ciudadanía substantiva y la gobernabilidad democrática en América Latina, con énfasis en el período que comienza en la década de 1970. Dado lo breve de este artículo introductorio, solamente presento aquí los lineamientos más genéricos de un argumento que he desarrollado en mayor detalle en otros trabajos (ver por ejemplo Castro, 2004, 2006, 2007, 2008, 2009). También por razones de espacio no trato en detalle aquí las características de los actores de las luchas por el agua, un tema que de todas maneras ha sido objeto de una amplia gama de trabajos que consideran, por ejemplo, el impacto de las identidades sociales de clase, étnicas o de género en las luchas sociales por el agua registradas en América Latina durante las últimas décadas (véase, por ejemplo, Laurie et. al., 2002; Bennett et. al., 2005; Crespo Flores, 2009; Castro y Costa Ribeiro, 2010). Finalmente, y por las mismas razones, este trabajo trata específicamente el caso de las luchas por el agua y no examina las interconexiones entre éstas y otros conflictos, aunque desde luego un estudio comprehensivo requeriría la consideración del entramado que componen las diversas formas de lucha social que tienen lugar en América Latina en relación con el control, propiedad y gestión del territorio (tierra, agua, bosques, etc.). Más bien, el centro de atención en este artículo son algunas distinciones

1 El original en inglés de este artículo fue publicado en Development, 2008, 51, pp. 72-76. Una traducción al español fue publicada previamente en: Anuario de Estudios Americanos, Vol. 66, No 2, 2009, pp. 15-22. 
analíticas que, en mi sugerencia, permiten avanzar en la sistematización del estudio de las luchas por el agua. Finalmente, cierro el trabajo con una breve referencia a las vinculaciones que pueden establecerse entre las luchas por el agua y el proceso de democratización en América Latina.

\section{Algunos patrones reconocibles en las luchas por el agua}

Una primera categorización de los eventos de lucha social por el agua identificados en América Latina desde la década de 1970 puede hacerse dividiéndolos en dos grandes grupos: luchas por el agua a) ecocéntricas y b) antropocéntricas. Como instrumento analítico esta distinción es en cierto modo artificial, ya que en la práctica la mayoría de los eventos de lucha por el agua tienden a combinar ambos aspectos. Sin embargo, la distinción no es enteramente arbitraria y además resulta de gran utilidad para iluminar ciertos aspectos específicos clave del proceso. En primer lugar, algunos expertos han insistido tradicionalmente en la necesidad de mantener una diferenciación analítica entre el estudio de los ecosistemas acuáticos, por una parte, y la infraestructura de los servicios derivados del agua o basados en ella. Por otra parte, la diferenciación se mantuvo, entre otras razones, porque históricamente ha existido una separación entre ambas áreas de actividad. Esto se ha reflejado, por ejemplo, en una distinción de estas dos esferas de acción en relación a los sistemas legales e institucionales que las regulan, las prácticas de gestión, e incluso en las formas que ha adoptado la división del trabajo entre las distintas disciplinas científicas que producen conocimiento sobre el agua, por ejemplo, entre la hidrología y la ingeniería de infraestructuras hídricas.

En segundo lugar, otra razón para mantener esta distinción analítica entre luchas ecocéntricas y antropocéntricas es que podemos identificar una cierta divergencia entre las luchas sociales conectadas con la protección de los ecosistemas acuáticos (por ejemplo, luchas contra la contaminación y el deterioro de dichos ecosistemas o contra los impactos negativos que la intrusión humana en la forma de grandes obras de infraestructura tiene tanto sobre el ciclo del agua como sobre la biodiversidad) y aquellas luchas orientadas a la defensa de los derechos de los seres humanos contemporáneos a 
un ambiente acuático limpio o al acceso a servicios de agua que son esenciales para la sobrevivencia y para el mantenimiento de condiciones de vida digna. Esta es una consideración importante porque las contradicciones que se dan al interior de los movimientos sociales y políticos conectados con las luchas por el agua a menudo expresan la existencia de intereses materiales, creencias, valores, principios y fines de carácter frecuentemente antagónico y, a veces, incluso irreconciliables. Aunque dichas contradicciones no sean necesariamente inevitables o irresolubles, sí es importante reconocer que las mismas son el resultado de procesos históricos específicos que caracterizan el desarrollo del movimiento ambientalista en general (véase, por ejemplo, Martínez Alier, 2002: 1-15), y que también se expresan en las contradicciones que pueden observarse entre los actores involucrados en las luchas por el agua en América Latina y en otros lugares.

Por ejemplo, muy frecuentemente los actores que están involucrados en luchas orientadas a la protección de ecosistemas acuáticos frágiles y de la vida silvestre acuática (por ejemplo, véase WWFLA, 2011) han mantenido silencio con respecto a la rápida expansión de la privatización del agua en esos mismos territorios, a pesar de las enormes consecuencias que dichas políticas tienen en relación a la gobernabilidad democrática y la justicia social. Lo opuesto también es cierto con frecuencia, ya que como tendencia los actores que han estado al frente de la lucha contra la privatización del agua han puesto menos énfasis sobre los problemas específicos vinculados a la necesidad de proteger y recuperar fuentes de agua que han sido históricamente objeto de una gestión ecológicamente deficiente por parte de agentes públicos y privados. Más aún, con frecuencia los actores que luchan por la defensa del acceso universal a los beneficios derivados de la gestión social del agua han tenido grandes dificultades para adoptar una posición crítica con respecto a las políticas gubernamentales fundadas en la construcción de infraestructuras hidráulicas de gran escala, como son las represas, las hidrovías o los transvases de cuencas, entre otros ejemplos, muchas de los cuales tienen impactos negativos tanto sobre los ecosistemas acuáticos como sobre las poblaciones humanas.

Esta última contradicción es un elemento crucial en los conflictos por el agua que afectan a muchos de los proyectos de desarrollo implementados 
en América Latina, en los cuales la construcción de grandes infraestructuras, con demasiada frecuencia, ha causado el desplazamiento forzado de poblaciones indígenas y de color, la expropiación autoritaria de derechos de agua sin compensación adecuada y daños irreversibles a los ecosistemas. Estas políticas han dado lugar, recurrentemente, a casos de corrupción público-privada y a la reproducción de formas de injusticia socio-ambiental en el acceso a los beneficios derivados de la construcción de dichas obras, trátese de proyectos de agua para consumo humano, para irrigación o para la protección de las poblaciones contra eventos extremos como son las sequías o las inundaciones (por ejemplo, véase Valencio, 1995; Boelens y Hoogendam, 2002).

Actualmente existe un gran número de obras de infraestructura hídrica en América Latina enmarañadas en luchas sociales surgidas de los impactos sociales, políticos, económicos, culturales y ambientales de estos proyectos, -las hidrovías, represas y otras obras- que son parte del Plan Puebla-Panamá en México y Centroamérica, y de la Iniciativa para la Integración de la Infraestructura Regional (IIRSA) en Sudamérica (véase, entre otras fuentes, MAB, 2011; OLCA, 2011, REDLAR, 2011, TRAGUA, 2011).

Una distinción analítica más fundamental, siempre en la perspectiva de este trabajo, lleva a diferenciar las confrontaciones entre las fuerzas socio-económicas y políticas que impulsan la expansión y consolidación de relaciones capitalistas, en particular, mediante la aceleración y extensión del proceso de mercantilización del agua, y las diferentes formas de resistencia contra el avance de formas hegemónicas de gestión y gobernabilidad del agua. Estas luchas de resistencia adoptan un amplio rango de estrategias y "vocabularios de protesta" (Guha y Martínez-Alier, 1997: 11-16), que van desde la defensa de los derechos indígenas y la denuncia del "racismo hídrico" hasta la recuperación de los bienes comunes y públicos y la búsqueda del reconocimiento de un "derecho humano" al agua. El análisis de este aspecto de las luchas por el agua requiere tomar distancia con respecto a los discursos y a los objetivos declarados de los actores y una consideración cuidadosa de las tendencias estructurales tanto como de la dirección que toman dichos procesos en última instancia ya que las dinámicas son, en gran medida, autónomas de las preocupaciones más inmediatas de los 
participantes. La comprensión de la relación entre la dimensión subjetiva de las luchas y las tendencias estructurales y dirección general del proceso está frecuentemente más allá del alcance de los actores, en gran medida debido al carácter multi-dimensional y trans-temporal de dicha dinámica. Por ejemplo, en los debates contemporáneos sobre la privatización del agua en América Latina frecuentemente se reifica la tensión entre lo "público" y lo "privado", lo cual tiende a obscurecer el hecho de que a pesar de las aparentes contradicciones entre agentes públicos y privados, ambos "bandos" contribuyen al avance y consolidación de formas capitalistas de gestión del agua fundadas en modelos de gobernabilidad democrática y ciudadanía meramente formales, no substantivos. Esto puede verse en la práctica, y no solamente en América Latina, en las reformas introducidas desde la década de 1980 en las empresas de agua y saneamiento de propiedad y gestión públicos. A partir de dichas reformas se espera que estas empresas presten servicios esenciales para la vida como los de agua y saneamiento sobre la base de principios mercantiles y se les exige que abandonen la noción de que dichos servicios constituyen un derecho social o un bien público, no digamos ya un derecho humano. Este proceso de transformación de las empresas públicas en entes que se comportan como empresas privadas cuyo objetivo prioritario es la producción de ganancia antes que la satisfacción de las necesidades esenciales de las poblaciones ya se encuentra muy avanzado en América Latina, incluyendo el caso de algunos países cuyos gobiernos se ubican nominalmente a la izquierda del espectro político. Esta situación es un ejemplo de que el proceso de expansión de consolidación de formas capitalistas de gestión y gobernabilidad del agua es en gran medida autónomo del carácter público o privado de los agentes involucrados.

En relación a lo anterior, cabe aclarar que la identificación de esta ambigüedad en las confrontaciones entre lo "público" y lo "privado" no significa que debamos perder de vista la distinción entre privatización (en sus diferentes formas) y gestión pública del agua y sus servicios. En este sentido, existen lecciones muy importantes derivadas de ejemplos excelentes identificados en América Latina, particularmente en Brasil, Venezuela, México, Uruguay, Argentina, El Salvador, Bolivia y Perú, entre otros casos, donde los actores han desarrollado una comprensión muy clara de estas complejidades: 
como tendencia general sus luchas contra la mercantilización del agua son dirigidas indistintamente contra iniciativas privadas o públicas. Esto se expresa, por ejemplo, en el hecho de que sus luchas combinan blancos múltiples: la oposición a la mercantilización de servicios esenciales de agua-que incluye la resistencia contra formas abiertas o disimuladas de privatización, como por ejemplo las asociaciones o partenariados público-privados o la mercantilización de las empresas públicas- intentos de revitalizar la noción de que dichos servicios constituyen un derecho social de ciudadanía, un bien común, un derecho humano tanto como estrategias dirigidas a fortalecer a las autoridades locales y desarrollar asociaciones o partenariados público-públicos para la gestión eficiente y transparente de los servicios esenciales de agua y saneamiento (véase, por ejemplo, Balanyá et. al., 2005; RED VIDA, 2011). Las iniciativas y prácticas de estos actores están dando lugar a formas emergentes, potencialmente emancipatorias, de gestión y gobernabilidad del agua que requieren apoyo para su profundización. Mi argumento es que dichas iniciativas forman parte de la lucha más amplia por la democratización substantiva en América Latina.

A pesar de lo breve, esta reseña de algunos aspectos centrales de las luchas por el agua en América Latina no puede dejar de mencionar algunos de los desafíos emergentes que confrontan la gestión y la gobernabilidad democráticas del agua en la región. Además de las tendencias mencionadas anteriormente, que incluyen el deterioro y la contaminación de las fuentes de agua dulce, los impactos negativos de las grandes obras de infraestructura y la expansión penetrante y consolidación de la mercantilización del agua en diferentes formas, quiero resaltar la existencia de dos fuentes recurrentes y potencialmente crecientes de confrontación por el agua, que en alguna medida se encuentran interrelacionadas. La primera de ellas tiene que ver con el impacto negativo de la rápida expansión de procesos productivos basados en usos intensivos del agua, como por ejemplo la minería y los cultivos masivos como son los del eucaliptus destinado a la producción de celulosa, o de la soja, la caña de azúcar, el maíz y otros cultivos, que se encuentran en el centro de la revolución de los agro-combustibles que está teniendo lugar en la región. Aunque el impacto de la minería sobre las fuentes y servicios esenciales de agua sí ha dado lugar a un intenso debate, en el caso de los 
cultivos masivos y especialmente en el de los agrocombustibles se ha sobre enfatizado el impacto potencial de estos procesos sobre la seguridad alimentaria, mientras que las implicaciones que los mismos tienen en relación a los ecosistemas acuáticos y a los servicios de agua han recibido mucha menos atención, a pesar de que ya son significativas en muchos lugares.

La segunda tendencia que quiero resaltar tiene que ver con las posibilidades de confrontaciones militares conectadas con el control y gestión del agua y otros recursos naturales, que ya es objeto de importantes discusiones. Aunque dichas posibilidades de conflicto militar han sido probablemente exageradas en algunos trabajos, lo cierto es que este tema ha pasado a ser un objeto de preocupación en la región, y especialmente en Sudamérica. Este es el caso, por ejemplo, del Acuífero Guaraní, el reservatorio de agua dulce subterráneo más grande del mundo que es compartido por Argentina, Brasil, Paraguay y Uruguay. Algunos gobiernos han desarrollado incluso hipótesis de guerra basadas en la posibilidad de que fuerzas militares extranjeras puedan intentar tomar control del acuífero. Si bien es posible que, en forma similar a otras hipótesis de guerra por el agua que se han postulado en otras partes del mundo, las bases reales para que se produzcan confrontaciones militares por el agua son mínimas. De todas formas, estas conjeturas reflejan la preocupación regional por la amenaza potencial de que el futuro de la gestión y la gobernabilidad del agua en América Latina se vea fuertemente marcado por una conflictividad creciente entre países. Por otra parte, este debate tiene también un lado oscuro: la discusión entre los gobiernos de la región sobre la posibilidad de confrontaciones militares internacionales por el agua podría contribuir a obscurecer la persistencia de conflictos intra-nacionales causados por la desigualdad y la injusticia en relación al agua.

\section{Conclusión: las luchas por el agua y el proceso de democratización en América Latina}

Como tendencia general la gestión y la gobernabilidad del agua en América Latina han sido caracterizadas históricamente, con pocas excepciones, por prácticas paternalistas y en gran medida verticales y excluyentes. 
La gobernabilidad del agua, en la perspectiva de este artículo, es un proceso político que involucra el ejercicio del poder por parte de actores sociales que buscan influir en la elección de los fines que deben orientar el proceso social, incluyendo los medios para lograr el alcance de dichos fines, en este caso en relación al control y gestión del agua y sus servicios y a la distribución de los beneficios (y la protección contra los peligros) derivados de los mismos. Siempre como tendencia general, este proceso ha sido caracterizado por el déficit democrático, si rechazamos la reducción del proceso democrático a la formalidad de los eventos electorales o su limitación al espacio de la política partidaria y, más bien, defendemos una noción de la democracia que reclama como principio de partida el ejercicio substantivo de la ciudadanía. Dadas estas tendencias no sorprende, entonces, que en muchos de los países de América Latina la historia de la gestión y la gobernabilidad del agua haya estado marcada por conflictos sociales, algunos de los cuales fueron agravándose desde mediados del siglo veinte debido al rápido avance de los procesos de urbanización e industrialización. Las políticas de privatización del agua y sus servicios desde la década de 1980 y la expansión generalizada de actividades productivas basadas en consumos intensivos de agua -que frecuentemente dependen de la explotación de ecosistemas acuáticos ya fragilizados-, han tendido a exacerbar los conflictos relacionados con el agua preexistentes y a promover la emergencia de nuevos conflictos. Estos eventos y tendencias, sin embargo, se han venido dando en un contexto de creciente estado de alerta en América Latina sobre las consecuencias ambientales del orden capitalista prevaleciente, el cual tiende a caracterizarse por la falta de atención a los procesos ecológicos cuando no directamente por prácticas que conducen a la destrucción ambiental. Ese estado de alerta creciente de la población en parte se expresa en las numerosas luchas por el agua que continúan deflagrándose en la región.

Si bien debe hacerse un esfuerzo para evitar proyectar visiones románticas de las luchas por el agua en América Latina, estoy convencido de que, y con relativa autonomía con respecto al nivel de conciencia de los actores y de sus objetivos declarados, dichas luchas están íntimamente conectadas con la lucha por la democratización substantiva de las sociedades de la región. En este sentido, una de las dimensiones cruciales de la lucha es el de 
la producción de conocimiento científico sobre el tema. En el caso del agua no hay alternativa a la visión radical del rol de la ciencia: se trata no solamente de estudiar la realidad sino de transformarla.

\section{Bibliografía}

Balanyá, Belén, Brid Brennan, Olivier Hoedeman, Satoko Kishimoto, y Philipp Terhorst (eds.) (2005), Reclaiming Public Water. Achievements, Struggles and Visions from Around the World, Amsterdam: Transnational Institute and Corporate Europe Observatory.

Bennett, Vivianne, Sonia Dávila-Poblete y María Nieves Rico (eds.) (2005), Opposing Currents The Politics of Water and Gender in Latin America, Pittsburgh: University of Pittsburgh Press.

Boelens, Rutgerd y Paul Hoogendam (eds.) (2002), Water Rights and Empowerment, Assen: Van Gorcum.

Castro, José Esteban y Wagner Costa Ribeiro (eds.) (2010), La Tensión entre Justicia Ambiental y Justicia Social en América Latina: el Caso de la Gestión del Agua, Ponencias de la Conferencia Internacional de la Red WATERLAT (107 trabajos), San Pablo y Newcastle upon Tyne (Reino Unido): Instituto de Estudios Avanzados de la Universidad de San Pablo, y Escuela de Geografía, Ciencia Política y Sociología de la Universidad de Newcastle.

Castro, José Esteban (2009), "Systemic conditions and public policy in the water and sanitation sector", en José Esteban Castro y Léo Heller (eds.), Water and Sanitation Services: Public Policy and Management, Londres y Sterling, VA.: Earthscan, pp. 19-37.

Castro, José Esteban (2008), "Los bienes comunes y la ciudadanía: contradicciones de una relación en pleno desarrollo", en Silke Helfrich (Coord.), Genes, Bytes y Emisiones: Bienes Comunes y Ciudadanía, México DF: Ediciones Fundación Heinrich Böll, pp. 68-80. 
Castro, José Esteban (2007), "Poverty and citizenship: sociological perspectives on water services and public-private participation", Geoforum, 38(5), pp. 756-771.

Castro, José Esteban (2006), Water, Power, and Citizenship. Social Struggle in the Basin of Mexico, Houndmills, Basingstoke and New York: Palgrave-Macmillan.

Castro, José Esteban (2004), "Urban water and the politics of citizenship: the case of the Mexico City Metropolitan Area (1980s-1990s)", Environment and Planning A, 36(2), pp. 327-46.

Crespo Flores, Carlos O. (2009), "Privatización del agua y racismo ambiental en ciudades segregadas. La empresa Aguas del Illimani en las ciudades de La Paz y El Alto (1997-2005)",

Anuario de Estudios Americanos, Vol. 66, No 2, pp. 105-122.

Guha, Ramachandra y Joan Martínez-Alier (1997), Varieties of Environmentalism: Essays North and South, Londres: Earthscan.

Laurie, Nina, Sarah Radcliffe, y Robert Andolina (2002), “The new excluded 'indigenous'?: The implications of multi-ethnic policies for water reform in Bolivia", en Rachel Sieder (ed.): Multiculturalism in Latin America. Indigenous Rights, Diversity and Democracy, Houndmills, Basingstoke y Nueva York: Palgrave-Macmillan, pp. 252-276.

Martínez-Alier, Joan (2002), The Environmentalism of the Poor. A Study of Ecological Conflicts and Valuation, Cheltenham y Northampton: Edward Elgar.

MAB - Movimento dos Atingidos por Barragens Brasil, 2011 (http://www. mabnacional.org.br, consultado el 27 de mayo de 2011). 
OLCA - Observatorio Latinoamericano de Conflictos Ambientales, 2011 (http://www.olca.cl, consultado el 27 de mayo de 2011).

REDLAR - La Red Latinoamericana contra Represas y por los Ríos, sus Comunidades y el Agua, 2011 (http://www.redlar.org/, consultado el 27 de mayo de 2011).

RED VIDA - Red de Vigilancia Interamericana para la Defensa y el Derecho al Agua, 2011 (http://www.laredvida.org/, consultado el 27 de mayo de 2011).

TRAGUA - Tribunal Latinoamericano del Agua, 2009 (http://www.tragua. com, consultado el 27 de mayo de 2011).

Valencio, Norma Felicidade (1995), Grandes Projetos Hidricos no Nordeste. Suas Implicaciones Para a Agricultura do Semi-arido, Natal, Brasil: Universidad Federal de Rio Grande do Norte.

WWFLA - World Wildlife Fund Latin America, 2011 (http://www.worldwildlife.org/wildplaces/lac.cfm, consultado el 27 de mayo de 2011). 